\title{
Blood pressure and hypertension in people living at high altitude in Nepal
}

\author{
Nirmal Aryal ${ }^{1}$, Mark Weatherall ${ }^{2}$, Yadav Kumar Deo Bhatta ${ }^{3}$, Stewart Mann $^{2}$
}

Authors details:

1. Faculty of Health \& Social Sciences, Bournemouth University, United Kingdom

2. Department of Medicine, University of Otago, Wellington, New Zealand

3. Department of Cardiology, Norvic International Hospital, Kathmandu, Nepal

\section{Corresponding Author}

Dr. Nirmal Aryal

Faculty of Health \& Social Sciences

Bournemouth University, United Kingdom

Royal London House (R313A), Christchurch Road, Bournemouth, BH1 3LT

Tel : +44 (0) 1202964329

Email: naryal@bournemouth.ac.uk

Running title: blood pressure in high altitude populations in Nepal

Funding: Capital Cardiovascular Research Trust, Wellington, New Zealand provided funding for the study as well as provided financial support for $\mathrm{PhD}$ study of $\mathrm{NA}$ at the University of Otago, Wellington, New Zealand. 


\begin{abstract}
This study aimed to describe blood pressure (BP) and hypertension (HT) in samples of high altitude populations of Nepal and to explore associations of systolic and diastolic BP with altitude. This was a cross-sectional survey of cardiovascular disease and associated risk factors among 521 people living at four different altitude levels, all above $2800 \mathrm{~m}$, in the Mustang and Humla districts of Nepal. Data on BP was available for all 521 participants. Systolic and diastolic BP levels were highest at the altitude of $3620 \mathrm{~m}$ (the highest area surveyed) but did not consistently increase with altitude. Using the cut-point of $\geq 140 / 90 \mathrm{mmHg}$ (systolic/diastolic), the prevalence of HT (or on anti-hypertensive medication) was $46.1 \%, 40.9 \%$, and $54.5 \%$ respectively at $2800 \mathrm{~m}, 3270 \mathrm{~m}$ and $3620 \mathrm{~m}$ of Mustang district, and $29.1 \%$ at $2890 \mathrm{~m}$ of Humla district. In a multivariate model adjusting for potential confounders there was strong evidence of a relationship between systolic BP and altitude; mean systolic BP increased by $15.6 \mathrm{mmHg}$ (95\% CI 4.0 to 27.2$), \mathrm{P}=0.009$ for every $1000 \mathrm{~m}$ elevation. Although diastolic BP and the probability for HT or on anti-hypertensive medication also tended to increase with increasing altitude levels, there was no evidence of a relationship. In the present study three out of four communities living at higher altitude levels showed a greater prevalence of HT among those aged 30 years or older compared with the overall national data. These findings indicate a probable high risk of raised BP in high altitude populations in Nepal.
\end{abstract}

Key words: adult, blood pressure, high altitude, hypertension, Nepal 


\section{Introduction}

The effect of chronic hypoxic hypobaric conditions, induced by living at high altitude (HA), on blood pressure (BP) is uncertain and may vary in different populations. Research on this relationship and other cardiopulmonary changes at HA has been ongoing for more than 50 years ${ }^{1}$, however, it is still uncertain whether the relationship between BP and hypertension (HT) and HA is causal, or a result of coincident lifestyle factors. An inverse association between altitude and systemic BP may be due to, separately or in combination with, structural changes in vasculature; and a number of socio-cultural, biological, chemical and physical factors. ${ }^{2,3}$ However, the possible benefit from altitude related hypoxia on systemic BP may diminish when genetic and lifestyle related risk factors become dominant, as seen in Tibetan origin populations. ${ }^{4,5}$

An altitude of 2500 metre (m) or above is the conventional demarcation for $\mathrm{HA}^{6}{ }^{6}$ however it is not uniformly defined in the scientific literature. Using this definition, more than 140 million people in the world permanently live at HA, comprising $2 \%$ of the global population. ${ }^{7}$ Established human populations living in the Ethiopian summits of Africa, the Himalayan mountains of Asia, and the Andean mountains of South America have a long history of HA residency: 70,000 years, 25,000 years, and 11,000 years respectively. ${ }^{8}$ The largest populations at HA are 80 million in the Himalayan mountains of Asia and 35 million in the Andean mountains of South America. ${ }^{7}$

In Nepal, almost 2 million people live permanently in mountainous areas, representing 7\% of the national population. ${ }^{9}$ A large proportion of the population of the hilly districts are also resident at altitudes greater than $2500 \mathrm{~m}$. According to earlier estimates, around $35 \%$ of the total Nepalese population permanently live at higher than $2500 \mathrm{~m}$ of elevation. ${ }^{7}$ Populations with Tibetan ancestry are the dominant HA population in Nepal.

There are few studies on HA living populations in Nepal. But none of them had information collected at different altitude levels above $2500 \mathrm{~m}$ and from ethnically diverse samples. This study aims to report the relationship between BP and altitude at four different altitude levels and tests the hypothesis 
that despite known hypoxia-induced favourable physiological responses on BP, HA residents in Nepal might have an increased risk of raised BP associated with lifestyle factors.

\section{Methods}

Study setting and sample

Two mountainous districts, Mustang and Humla, were selected for sampling based on past documentation of geography, altitude, population and ethnicity and the feasibility for conducting the study. There are 16 districts classified as mountain ecological regions in Nepal. The selected study areas were, in Mustang district, urban settings of Jomsom (2800 m) and Muktinath (3620 m), and a rural setting of Jharkot $(3270 \mathrm{~m})$; and in Humla district, a rural setting of Simikot $(2890 \mathrm{~m})$. The population with Tibetan ancestry is predominant in Mustang district, whereas Khas-Aryan population has a majority in Humla. Mustang people are relatively wealthy and the dominant Thakali ethnic group has the highest human development index (HDI) score in $\mathrm{Nepal}^{10}$ which is largely due to tourism and natural resources. In contrast, the Humla district has the lowest HDI score ${ }^{10}$. Tibetan culture is influential in both these districts because both are bordered with Tibetan sides of China. Life expectancy at birth is 64.1 years in Mustang and 65.0 years in Humla, both lower than the national figure of 68.8 years $^{10}$.

Tibetans migrated to high mountains of Nepal about 500 years ago. Tibeto-Burman is their native language and they practice Buddhism ${ }^{11}$. Thakali, Tibetan Gurung, and Lama of the study's population belong to this category. Khas-Aryans migrated to Nepal about 2000 years ago, speak the Nepalese language, and the majority of them are Hindu ${ }^{12}$. Brahmins, Chettris, and Dalits are mainly categorized as Khas-Arya ${ }^{13}$. Tibetans and Khas-Aryans have distinctive physical appearances, languages, and names.

The inclusion criteria for study participants were age 30 years and above and the ability to speak and understand the Nepalese language. Exclusion criteria were the inability or unwillingness to provide written or verbal consent, inability to speak or hear properly, and pregnancy. 


\section{Sampling process}

Sampling was carried out in two stages. In the first stage, three study areas of Mustang and one study area of Humla were selected on the basis of altitude levels, population density and logistical support to undertake the study. In the second stage, a list of households was developed, and a unique number was assigned to each of them. Household numbers were randomly selected with the help of a computer based randomization technique. All eligible family members of the randomly selected households were considered. If none of the household members were eligible or agreed to take part in the study, a household in the close proximity was selected.

The desired sample size within each district was calculated to be around 250 individuals. This sample size gave a margin of error of plus or minus $5 \%$ for a proportion based on anticipated prevalence of HT of around 25\% after adjusting for the design effect and an expected non-response rate of $10 \%$.

\section{Data collection procedures}

The World Health Organization's STEPS questionnaire version 2.2 for non-communicable disease risk factors was administered to consenting participants. This questionnaire has three sections. The first section has questions on socio-demographic and lifestyle factors, a second section consists of BP and anthropometric (height, weight, waist, hip) measurements, and the third section contains measurements of blood glucose and lipids. The Nepal Health Research Council (NHRC) had already translated this into the Nepalese language and it has been validated through a pilot study in a community and approved by expert meetings ${ }^{14}$.

Administration of the questionnaire and BP measurements were carried out at the house of the selected participants. Practically it was not feasible to undertake blood sample testing for lipid components and glycated hemoglobin ( $\mathrm{HbA1c}$ ), and bio-physical measurements in individual houses so a local community hall was used as the measurement facility. 
Data was collected between June and August (summer) 2014 in Mustang district and between March and May (spring) 2015 in Humla district. The ambient temperature was between $25^{\circ} \mathrm{C}$ and $31^{\circ} \mathrm{C}$ in Mustang and around $15^{\circ} \mathrm{C}$ in Humla on most of study days.

BP was measured by oscillometric method using an automatic blood pressure measuring device (Omron HEM-7221). The recommended operating ambient pressure conditions of this machine are from 700 to 1060 hectopascals. This is the atmospheric pressure at around $3000 \mathrm{~m}$. Moreover, Omron BP monitoring machines have also passed the validation criteria at $3650 \mathrm{~m}$ as proposed by the European Society of Hypertension International Protocol Revision 2010 (ESH-IP2) ${ }^{15}$. Three readings were recorded from left arm of each participant in sitting position with at least 10 minutes elapsing in between. The first measurement was taken a minimum of 10 minutes after the questionnaire was administered. If the readings differed by more than 10 units, a fourth measurement was taken and the lowest three were considered for an average calculation. HbA1c and lipid profiles were measured using a Cobas b 101 device (Roche Diagnostics). A small amount of capillary whole blood was taken by single finger-prick and processed by the device.

\section{Definitions}

Blood pressure (systolic/diastolic) was classified as normal $(<120 / 80 \mathrm{mmHg})$, pre-HT $(120-39 / 80-89$ mmHg), HT ( $\geq 140 / 90 \mathrm{mmHg})$, Stage I HT (140-159/90-99 mmHg), and Stage II HT $(\geq 160 / 100$ $\mathrm{mmHg}$ ) according to the seventh report of the Joint National Committee on prevention, detection, evaluation, and treatment of high blood pressure ${ }^{16}$. Alcohol consumption-related questions were about current, past and 'ever drinking' status, frequency of drinking, and the number of standard drink units (SDUs) consumed in the past 30 days. Current drinkers were defined as those drinking alcohol in the past 30 days. SDU of alcohol was calculated by the formula:

Amount of drink (Litre)(l) $x$ percent of volume of alcohol (\%) $x$ density of ethanol at room temperature $(0.789)^{17}$.

A glass with 90 millilitre $(\mathrm{mL})$ volume was a popular size in the study areas. The few participants who consumed alcohol in different-sized glasses were asked to estimate the volume with reference to 
a $90 \mathrm{~mL}$ glass. Traditionally home-made and locally brewed alcoholic beverages were popular in the study areas, mainly jaad (made of rice), chang (also made of rice but using a different method), and raksi (made of rice, millet or barley). The percentage of volume of alcohol was determined as $12 \%$ for jaad and chang, $25 \%$ for raksi ${ }^{18}$, and $5.5 \%$ for beer and $40 \%$ for vodka as labelled.

The amount of salt consumption was determined by asking how much participants estimated their household consumed in a month, and this amount was divided by the number of family members currently sharing the kitchen for the main meal. Salt was usually available in one kilogram $(\mathrm{kg})$ packets.

\section{Statistical methods}

Simple data description is by mean, standard deviation (SD), frequency counts, and proportions expressed as percentages. The Clopper-Pearson method was used to estimate an exact confidence interval for a single proportion. All results are presented for each level of altitude. Analysis of variance (ANOVA) and analysis of covariance (ANCOVA) models for the relationship between systolic blood pressure (SBP), diastolic blood pressure (DBP) and altitude are shown. Logistic regression was used to estimate the association between HT or on antihypertensive medication and altitude. Altitude was treated as a continuous variable in the multivariate models. Other included variables in multivariate models were age, sex, weight, salt intake, alcohol consumption (SDUs) in the past 30 days, urban or rural residential setting, and current smoking status. Stata version 12 was used for data analysis.

This study was approved by the ethical review board of NHRC and the University of Otago, Human Ethics Committee. 


\section{Results}

A total of $521 \mathrm{HA}$ residents at four different altitude levels participated in this study; $2800 \mathrm{~m}$ $(\mathrm{N}=165), 3270 \mathrm{~m}(\mathrm{~N}=61)$ and $3620 \mathrm{~m}(\mathrm{~N}=44)$ of the Mustang district and $2890 \mathrm{~m}(\mathrm{~N}=251)$ of the Humla district. More than $70 \%$ of participants in Mustang had Tibetan ancestry and $>70 \%$ in Humla were Khas-Aryans. Particular ethnic groups amongst Tibetan related participants were: Thakali, Tibetan Gurung and Lama; and for Khas-Aryans: Brahmin, Chhetri, and Dalit. Participants are described in Table 1.

Figure 1 shows boxplots of SBP and DBP by the levels of altitude. The mean SBP and DBP were highest at the highest altitude of $3620 \mathrm{~m}$ but did not consistently increase with increasing altitude. There was strong evidence that SBP and DBP were different across the altitude levels. The F-statistics from the analysis of variance (ANOVA) with $\mathrm{SBP}$ as the response variable and altitude as a categorical predictor variable was 12.6 (3 DF, 517), P <0.01; and for DBP, 3.64 (3 DF, 517), P=0.01.

Summaries of HT-related characteristics at different altitude levels are shown in Table 2. Residents at $3620 \mathrm{~m}$ had higher proportions of participants with HT and related characteristics than those at lower altitude. Participants who were men, in older age groups, with formal school education, higher selfreported annual household income and currently married were more likely to have HT (or be on antihypertensive medication). Men had higher levels of SBP and DBP at each altitude level compared with women, however, this differences were statistically significant at $2890 \mathrm{~m}$ only, for both SBP and DBP. Overall, men had almost two-fold higher odds than women for being hypertensive or on antihypertensive medication: age adjusted odds ratio (95\% CI) of 1.96 (1.34 to 2.86$), \mathrm{P}<0.01$.

By self-report about half of the participants at all altitude stated they were current consumers of alcohol. A higher proportion of men were current drinkers compared to women at every altitude level. At least a quarter of the current drinkers or current smokers were hypertensive or on anti-hypertensive medication at all altitude levels. There were no significant differences between currently smoking status, current consumers of alcohol and having hypertension (or on anti-hypertensive medication) at any altitude level. Hypertensive residents at all altitude levels usually reported that on average they 
consumed less fruit and vegetables and more salt per day compared to non-hypertensives, however, the difference was statistically significant only at $2800 \mathrm{~m}$ for average fruit and vegetables consumption only. Although differences were statistically non-significant, those with HT (or on antihypertensive medication) were generally physically less active and sedentary at all altitude levels compared to non-hypertensives.

Participants with Tibetan ethnicity had a higher proportion of HT (or being on anti-hypertensive medication) compared to Khas-Aryans; however, when adjusted for age and sex the odds ratio (95\% CI) for HT (or being on anti-hypertensive medication) among participants with Tibetan compared to Khas-Aryan was 0.86 (0.58 to 1.29$), \mathrm{P}=0.49$.

In a multivariate model adjusting for potential confounders there was strong evidence of a relationship between SBP and altitude (Table 3). Mean SBP (95\% CI) increased by $15.6 \mathrm{mmHg}$ (4.0 to 27.2), $\mathrm{P}=0.009$ for every $1000 \mathrm{~m}$ elevation. Older age, self-reported SDUs of alcohol in the past 30 days, and being urban residents were associated with higher mean SBP.

Table 4 shows the estimates of the associations between DBP and altitude and other covariates. In the multivariate model adjusting for the potential confounders, the relationship between DBP and altitude was positive, albeit with wide confidence interval. Mean (95\% CI) DBP increased by $7.2 \mathrm{mmHg}(-0.2$ to 14.6$), \mathrm{P}=0.05$ per $1000 \mathrm{~m}$ higher altitude. Among the covariates, higher weight and a greater number of SDUs of alcohol in the past 30 days was associated with higher DBP. Unexpectedly, there was a negative relationship of self-reported salt intake with both mean SBP and DBP, however these did not reach the level of statistical significance.

While adjusting for the same confounding variables as for mean SBP and DBP, the odds ratio (95\% CI) for being hypertensive or on anti-hypertensive medication was 2.21 ( 0.55 to 8.96$), \mathrm{P}=0.26$ per $1000 \mathrm{~m}$ higher altitude. Among the covariates, only older age was significantly associated with the probability for having hypertension or on anti-hypertensive medication. 


\section{Discussion}

The present study found a strong association between SBP and altitude. Although point estimates for the associations between DBP and HT (or on anti-hypertensive medication) and increasing altitude levels were consistent with a positive association, confidence intervals for the estimates of associations were wide. This study did not identify any association between HT (or use of antihypertensive medication) and ethnicity (categorized as Tibetan related or Khas-Aryan). Men were more likely to have higher SBP and DBP than women at every altitude level.

A recent systematic review reported a weak positive association between SBP, DBP and altitude in populations of Tibetan origin but in non-Tibetan populations both SBP and DBP tended to decrease with residence at higher altitude, although there were wide confidence intervals ${ }^{19}$. Another systematic review including studies conducted in Tibet reported a $2 \%$ increment in the prevalence of HT for every $100 \mathrm{~m}$ increment in altitude ${ }^{5}$. These findings are consistent with findings in the present study where, although participants were from both Tibetan and Khas-Aryan ethnicities, the geographical location of the study districts border on the Tibetan side of China and Khas-Aryans have consequently acquired Tibetan lifestyle practices, mainly drinking tea made with salty butter and high amounts of alcohol. In the present study, approximately half of the participants at all altitude levels self-reported being current drinkers, which is greater than the national prevalence of $21.5 \%$ for the Nepalese population aged older than 30 years $^{14}$. This may well contribute to the association between SBP and altitude regardless of ethnicity. Nonetheless, the association between altitude and SBP was found even after controlling for alcohol and salt intake, which may indicate that the HA environment itself is an additional independent factor in determining the high prevalence of HT although there may also be an underlying genetic risk ${ }^{4,20}$.

The lack of significant association of DBP with altitude - although this association was positive may be partly due to the significant number of participants aged 60 years or above (around one-fifth) and it is well established that DBP falls with ageing while SBP continues to rise ${ }^{21}$. Although the present study's finding of multivariate adjusted associations between BP and age, alcohol intake, and 
urban lifestyle is consistent with past research the inverse relationship between self-reported daily salt consumption and $\mathrm{BP}$ is not ${ }^{22,23}$. High consumption of salt as a traditional dietary habit has been identified as one of the key factors for high BP in HA Tibetan populations ${ }^{24,25}$. The method used in the present study of self-report to calculate salt consumption could have systematically led to underestimation of salt intake in those with the highest salt intake.

The prevalence of HT (or those on anti-hypertensive medication) in participants in the present study is higher than in other studies carried out in HA populations in Nepal using a similar definition ${ }^{26-28}$. Two possible explanations for this difference are the different ages of the study samples and a cohort effect. The minimum age to participate in the present study was 30 years, whereas other studies usually included participants from the age of 18 years or over. HT may be increasing in prevalence with the passage of time for reasons other than lifestyle, ethnicity or altitude. However, in the present study, the prevalence of HT (or those on anti-hypertensive medication) in the Mustang district (Tibetan related participants), is close to that reported in HA studies carried out on Tibetans living in Tibet or in India ${ }^{29-31}$. The prevalence of HT (or being on anti-hypertensive medication) in Mustang was higher than the national prevalence of Nepal for the population aged 30 years or over $(36.8 \%)^{14}$, but was lower in the Humla district with its dominant Khas-Aryan population. In the present study the prevalence of raised BP was high, but actual diagnosis and treatment of HT was very poor. Nearly one-quarter of the study participants and two-thirds of hypertensives were diagnosed with HT for the first time during the study.

The main strength of the present study for the relationship between BP and HA is that presumably this is the first study to collect information at four different altitude levels above or equal to $2500 \mathrm{~m}$ and from ethnically diverse samples. The present study has several limitations. Multiple potential confounding and effect modification factors could explain an apparent relationship between SBP and altitude; mainly genetic risk, cultural-related lifestyle practices, socio-economic status, and residential settings (rural versus urban). These differences are accounted for where relevant data were available but these inter-relationships are complex. Other limitations are: selection of the study sites on the basis of logistic convenience for the study conduct, low population density and consequently a low 
sample size at $3270 \mathrm{~m}$ and $3620 \mathrm{~m}$ of Mustang district, reliance on self-report in an area of poor health literacy and the cross-sectional design. There is also the risk of 'healthy individual effect' because, in the absence of quality health care, people with HT might have moved to low altitude areas for treatment or died, so reported prevalence might not reflect true incidence. On the other hand, it is also likely that healthy young populations of study areas may have internally migrated to city areas or emigrated to other countries for job, education or for other reasons. Thus, this 'migration effect' could have missed eligible young and healthy individuals in the survey. Finally, there was a notable difference in the ambient temperature of two study districts at the time of data collection. Temperature of Humla was lower by between $10{ }^{\circ} \mathrm{C}$ to $16^{\circ} \mathrm{C}$ than that of Mustang. As there is some evidence that lower ambient temperature increases BP in adults ${ }^{32}$, difference of temperature in study districts might have modified the effect of altitude on BP.

In conclusion, there was a strong evidence of a relationship between SBP and altitude, and no evidence of a relationship between DBP, HT and altitude, after adjustment for potential confounding variables. The prevalence of HT was higher in three out of four altitude levels compared to the national prevalence of Nepal for populations aged 30 years or older. These findings indicate a probable high risk of raised BP in HA populations in Nepal and clinicians should be aware about it. Furthermore, the present study also observed that the non-Tibetan HA population of Nepal has acquired Tibetan lifestyle habits, which may increase the risk of HT in the future. Public health awareness on healthier lifestyle as well as clinical intervention campaign for diagnosis, treatment, and control of HT are recommended in these HA populations. There is very limited research about BP among the non-Tibetan HA populations in Nepal. More data and analysis of these populations will help to better understand the HA and BP relationship. Although high intake of salt and heavy alcohol drinking are mainly attributed for raised BP in HA populations of Nepal, information on uptake levels by objective measures is limited. Comparisons of HA populations with homogeneous counterparts who later migrated to low altitude may also provide important insights into this relationship. 
Conflict of Interest : The authors declare no conflict of interest.

Acknowledgments: We would like to specially thank research assistants Prakash Pant, Ashish Khadayat and Mamata Chand for their incredible support in data collection. We are grateful to local nurses Sofia Shrestha, Reena Thakali, and female community health volunteers Lalita Sherchan (Mustang) and Guyala Rawat (Humla) who helped significantly in various aspects of data collection.

Funding: Capital Cardiovascular Research Trust, Wellington, New Zealand provided funding for the study as well as provided financial support for $\mathrm{PhD}$ study of NA at the University of Otago, Wellington, New Zealand.

Author's Contribution: All authors contributed to study design. NA collected the data, performed statistical analysis and prepared the first draft of the manuscript. YKDB helped with data collection and MW helped with the statistical analysis. SM provided overall guidance and supervision. All authors contributed intellectually to the revision of the article and approved the final version. 


\section{References}

1. Ostadal B, Kolar F. Cardiac adaptation to chronic high-altitude hypoxia: beneficial and adverse effects. Respir Physiol Neurobiol 2007; 158: 224-236.

2. Parati G, Ochoa JE, Torlasco C, Salvi P, Lombardi C, Bilo G.Aging, high altitude, and blood pressure: a complex relationship. High Alt Med Biol 2015; 16: 97-109.

3. Ruiz L, Penaloza D. Altitude and hypertension. Mayo Clin Proc 1977; 52: 442-445.

4. Gesang L, Liu G, Cen W, Qiu C, Zhuoma C, Zhuang L, Ren D, Pincuo Z, Chan Y. Angiotensin-converting enzyme gene polymorphism and its association with essential hypertension in a Tibetan population. Hypertens Res 2002; 25: 481-485.

5. Mingji C, Onakpoya IJ, Perera R, Ward AM, Heneghan CJ. Relationship between altitude and the prevalence of hypertension in Tibet: a systematic review. Heart 2015: heartjnl-2014307158. doi: 10.1136/heartjnl-2014-307158.

6. Pawson I, Jest C. The high-altitude areas of the world and their cultures. The biology of highaltitude peoples 1978: 17-45.

7. Moore LG, Niermeyer S, Zamudio S. Human adaptation to high altitude: Regional and lifecycle perspectives. Am J Phys Anthropol 1998; 107: 25-64.

8. Beall CM. Two routes to functional adaptation: Tibetan and Andean high-altitude natives. Proceedings of the National Academy of Sciences 2007; 104: 8655-8660.

9. Nepal in Figures. Government of Nepal, National Planning Commission Secretariat; 2012. http://cbs.gov.np/wp-

content/uploads/2012/Nepal\%20in\%20figure/Nepal\%20In\%20Figures\%202012_English.pdf. Accessed 13th September, 2013.

10. Government of Nepal \& United Nations Development Programme. Nepal human development report 2014: beyond geography, unlocking human potential. Kathmandu, Nepal2014.

11. Rambal C. Tibetan pride of place : or, why Nepal's Bhotiyas are not an ethnic group. In: Gellner DN, Pfaff-Czarnecka J, Whelpton J, eds, Nationalism and ethnicity in a Hindu Kingdom: the politics of culture in contemporary Nepal. Taylor and Francis: New York, 2012, 379-414.

12. Eagle S. The language situation in Nepal. In: Baldauf RB, Kaplan RB, eds, Language Planning in Nepal, Taiwan, and Sweden, Vol 20. Multilingual Matters Ltd.: Clevedon, UK, 2000.

13. Gellner DN. The idea of Nepal. Social Science Baha: Kathmandu, 2016.

14. Aryal KK, Mehata S, Neupane S, Vaidya A, Dhimal M, Dhakal P, Rana S, Bhusal CL, Lohani GR, Paulin FH, Garg RM. The burden and determinants of non-communicable diseases risk factors in Nepal: findings from a nationwide STEPS Survey. PloS one 2015; 10: e0134834. doi: 10.1371/journal.pone.0134834.

15. Cho K, Tian M, Lan Y, Zhao X, Yan LL. Validation of the Omron HEM-7201 upper arm blood pressure monitor, for self-measurement in a high-altitude environment, according to the European Society of Hypertension International Protocol revision 2010. J Hum Hypertens 2013; 27: 487-491.

16. Chobanian AV, Bakris GL, Black HR, Cushman WC, Green LA, Izzo Jr JL, Jones DW, Materson BJ, Oparil S, Wright Jr JT, Rocella EJ and the National High Blood Pressure Education Program Coordinating Committee. Seventh report of the joint national committee on prevention, detection, evaluation, and treatment of high blood pressure. Hypertension 2003; 42: 1206-1252.

17. Health Promotion Agency New Zealand. What's a standard drink ? 2015; http://alcohol.org.nz/help-advice/standard-drinks/whats-a-standard-drink. Accessed 12th May 2018.

18. Pradhan B, Chappuis F, Baral D, Karki P, Rijal S, Hadengue A, Gache P. The alcohol use disorders identification test (AUDIT): validation of a Nepali version for the detection of alcohol use disorders and hazardous drinking in medical settings. Subst Abuse Treat Prev Policy 2012; 7: 42. 
19. Aryal N, Weatherall M, Bhatta YKD, Mann S. Blood pressure and hypertension in adults permanently living at high altitude : a systematic review and meta-analysis. High Alt Med Biol 2016; 17: 185-193.

20. Kumar R, Pasha MQ, Khan AP, Gupta V, Grover SK, Norboo T, Srivastava KK, Selvamurthy W, Brahamchari SK. Association of high-altitude systemic hypertension with the deletion allele-of the angiotensin-converting enzyme (ACE) gene. Int J Biometeorol 2003; 48: 10-14.

21. Burt VL, Whelton P, Roccella EJ, Brown C, Cutler JA, Higgins M, Horan MJ, Lebarthe D. Prevalence of hypertension in the US adult population. Hypertension 1995; 25: 305-313.

22. Elliott P, Stamler J, Nichols R, Dyer AR, Stamler R, Kesteloot H, Marmot M. Intersalt revisited: further analyses of 24 hour sodium excretion and blood pressure within and across populations. BMJ 1996; 312: 1249-1253.

23. Pietinen P, Uusitalo U, Nissinen A. Intersalt: an international study of electrolyte excretion and blood pressure. Results for 24 hour urinary sodium and potassium excretion. BMJ 1988.

24. Sun S. Epidemiology of hypertension on the Tibetan Plateau. Hum Biol 1986: 507-515.

25. Sehgal A, Krishan I, Malhotra R, Gupta H. Observations on the blood pressure of Tibetans. Circulation 1968; 37: 36-44.

26. Smith C. Blood pressures of Sherpa men in modernizing Nepal. Am J Hum Biol 1999; 11: 469-479.

27. Koju R, Manandhar K, Risal A, Steiner T, Holen A, Linde M. Undertreated Hypertension and its Implications for Public Health in Nepal: Nationwide Population-Based Survey. Kathmandu Univ Med J 2015; 13: 3-7.

28. Shrestha S, Shrestha A, Shrestha S, Bhattarai D. Blood Pressure in Inhabitants of High Altitude of Western Nepal. J Nepal Med Assoc 2012; 52: 154-158.

29. Zhao X, Li S, Ba S, He F, Li N, Ke L, Li X, Lam C, Yan LL, Zhou Y, Wu Y. Prevalence, awareness, treatment, and control of hypertension among herdsmen living at 4,300 $\mathrm{m}$ in Tibet. Am J Hypertens 2012; 25: 583-589.

30. Zheng X, Yao D-K, Zhuo-Ma C-R, Tang J, Wang TR, Zhang HH, Wang LX. Prevalence, self-awareness, treatment, and control of hypertension in Lhasa, Tibet. Clin Exp Hypertens 2012; 34: 328-333.

31. Tripathy V, Gupta R. Blood pressure variation among Tibetans at different altitudes. Ann Hum Biol 2007; 34: 470-483.

32. Wang Q, Li C, Guo Y, Barnett AG, Tong S, Phung D, Chu C, Dear K, Wang X, Huang C. Environmental ambient temperature and blood pressure in adults: A systematic review and meta-analysis. Sci Total Environ 2017; 575: 276-286. 
Figure 1: Box plot of systolic and diastolic blood pressure values by altitude level 